\title{
Crystalline monolayer surface of liquid Au-Cu-Si- Ag-Pd: Metallic glass former
}

\section{Citation}

Mechler, S., E. Yahel, P. S. Pershan, M. Meron, and B. Lin. 2011. “Crystalline Monolayer Surface of Liquid Au-Cu-Si-Ag-Pd: Metallic Glass Former.” Appl. Phys. Lett. 98 (25): 251915.

\section{Published Version}

doi:10.1063/1.3599515

\section{Permanent link}

http://nrs.harvard.edu/urn-3:HUL.InstRepos:17223815

\section{Terms of Use}

This article was downloaded from Harvard University's DASH repository, and is made available under the terms and conditions applicable to Other Posted Material, as set forth at http:// nrs.harvard.edu/urn-3:HUL.InstRepos:dash.current.terms-of-use\#LAA

\section{Share Your Story}

The Harvard community has made this article openly available.

Please share how this access benefits you. Submit a story.

\section{Accessibility}




\title{
Crystalline monolayer surface of liquid $\mathrm{Au}-\mathrm{Cu}-\mathrm{Si}-\mathrm{Ag}-\mathrm{Pd}$ : Metallic glass former
}

\author{
S. Mechler, ${ }^{1, a)}$ E. Yahel, ${ }^{1,2}$ P. S. Pershan, ${ }^{1}$ M. Meron, ${ }^{3}$ and B. Lin $^{3}$ \\ ${ }^{1}$ Department of Physics and SEAS, Harvard University, Cambridge, Massachusetts 02138, USA \\ ${ }^{2}$ Department of Physics, NRCN, Be'er-Sheva 84190, Israel \\ ${ }^{3}$ CARS, University of Chicago, Chicago, Illinois 60637, USA
}

(Received 13 March 2011; accepted 22 May 2011; published online 24 June 2011)

\begin{abstract}
It is demonstrated by means of $\mathrm{x}$-ray synchrotron reflectivity and diffraction that the surface of the liquid phase of the bulk metallic glass forming alloy $\mathrm{Au}_{49} \mathrm{Cu}_{26.9} \mathrm{Si}_{16.3} \mathrm{Ag}_{5.5} \mathrm{Pd}_{2.3}$ consists of a two-dimensional crystalline monolayer phase for temperatures of up to about $50 \mathrm{~K}$ above the eutectic temperature. The present alloy as well as glass forming $\mathrm{Au}_{82} \mathrm{Si}_{18}$ and $\mathrm{Au}-\mathrm{Si}-\mathrm{Ge}$ alloys containing small amounts of $\mathrm{Ge}$ are the only metallic liquids to exhibit surface freezing well above the melting temperature. This suggests that the phenomena of surface freezing in metallic liquids and glass forming ability are related and probably governed by similar physical properties. (C) 2011 American Institute of Physics. [doi:10.1063/1.3599515]
\end{abstract}

Over the past two decades, the surface structure of more than a dozen liquid metals and alloys has been studied. Although every one of these systems exhibits surface induced atomic layering that was proposed by D'Evelyn and Rice in $1981{ }^{1}$ the only eutectic alloys that form two-dimensional (2D) surface crystals at temperatures well above the eutectic melting temperature $\left(T_{e}\right)$ are the $\mathrm{Au}_{82} \mathrm{Si}_{18}$ eutectic ${ }^{2-6}$ and ternary $\mathrm{Au}-\mathrm{Si}-\mathrm{Ge}$ eutectics containing up to about 2.8 at. \% Ge. ${ }^{7}$ On the other hand, there are other examples of surface freezing in which a $2 \mathrm{D}$ crystalline phase forms within a surface segregated monolayer of liquid Ga alloys containing low concentrations of Pb or TI. ${ }^{8,9}$ The Ga systems are different in that the 2D crystals only form at coexistence with bulk $\mathrm{Pb}$ or TI. In contrast the low temperature (LT) $2 \mathrm{D}$ crystalline bilayer surface phase of $\mathrm{Au}_{82} \mathrm{Si}_{18}$ that forms on melting persists for $12 \mathrm{~K}$ above $T_{e}$, at which point it undergoes a firstorder phase transformation to a $2 \mathrm{D}$ crystalline monolayer high temperature (HT) phase. ${ }^{6}$ At about $60 \mathrm{~K}$ above $T_{e}$ the HT crystals melt into a liquidlike (LL) surface that exhibits the same kind of atomic layering normal to the surface that is found for all other liquid metals. However, the electron density model that best fits the reflectivity for the LL phase of $\mathrm{Au}_{82} \mathrm{Si}_{18}$ is nearly as well defined as that of a crystalline surface. ${ }^{6}$ The implication is that the atomic layering nearest the surface of $\mathrm{Au}_{82} \mathrm{Si}_{18}$ is much more pronounced than for other liquid metals. The origin of $2 \mathrm{D}$ surface crystalline order of liquid $\mathrm{Au}_{82} \mathrm{Si}_{18}$ is still not understood. Surprisingly, the closely related Au-based eutectic $\mathrm{Au}_{72} \mathrm{Ge}_{28}$ alloy does not display surface freezing and the layering of the liquidlike surface is much weaker than in $\mathrm{Au}_{82} \mathrm{Si}_{18}$.

Historically, $\mathrm{Au}_{75} \mathrm{Si}_{25}$, an alloy slightly off of the eutectic composition of $\mathrm{Au}_{82} \mathrm{Si}_{18}$, was the first alloy that was successfully quenched from the liquid phase into an amorphous, solid phase. ${ }^{11}$ This finding stimulated intense research on glass formation in metallic alloys and over the last decades a large number of systems have been discovered to have exceptional glass forming ability. Many of these have potential for use as engineering materials, e.g., Zr-, Pd-, or Au-based bulk metallic glasses. ${ }^{12}$ While the microscopic origin of glass

${ }^{\text {a)} E l e c t r o n i c ~ m a i l: ~ s m e c h l e r @ s e a s . h a r v a r d . e d u . ~}$ formation is still intensively debated, ${ }^{13-15}$ alloy systems with good glass forming ability generally seem to be characterized by a composition near a deep eutectic, large differences in the atomic sizes of the components as well as a large negative heat of mixing between the components. As a consequence of this, glass forming liquids exhibit a high degree of chemical and topological short range order (SRO) in the liquid phase. This SRO often appears in the form of an icosahedral SRO and/or medium range order in the liquid and the resulting amorphous phase..$^{13,14,16,17}$ It was suggested that this icosahedral order inhibits formation of bulk crystalline phases during undercooling/quenching. ${ }^{18}$

In addition, the binary Au-Ge alloy cannot be quenched into an amorphous phase, ${ }^{19}$ although it otherwise has similar properties than $\mathrm{Au}-\mathrm{Si}$. This suggests that surface freezing in the liquid phase and glass formation might have a common origin. In this letter we present $\mathrm{x}$-ray synchrotron studies of the surface properties of the liquid phase of the $\mathrm{Au}_{49} \mathrm{Cu}_{26.9} \mathrm{Si}_{16.3} \mathrm{Ag}_{5.5} \mathrm{Pd}_{2.3}$ metallic alloy that is known to have a very high glass forming ability. ${ }^{20}$ The $\mathrm{x}$-ray results reveal the formation of a $2 \mathrm{D}$ monolayer crystalline surface phase for temperatures up to $50 \mathrm{~K}$ above the eutectic temperature.

The $\mathrm{Au}_{49} \mathrm{Cu}_{26.9} \mathrm{Si}_{16.3} \mathrm{Ag}_{5.5} \mathrm{Pd}_{2.3}$ liquid sample with a eutectic temperature of $T_{e} \approx 625 \mathrm{~K}$ (Ref. 20) was prepared by melting its components (with purity $\geq 99.99$ at. \%) in a fully dense, high purity $\mathrm{Al}_{2} \mathrm{O}_{3}$ ceramic crucible (additional experimental details are described in Ref. 6. The accuracy in the absolute temperature measurement is estimated to be around $\pm 5 \mathrm{~K}$, while the accuracy of relative temperature differences is better than $\pm 1 \mathrm{~K}$. Experiments were performed at the $\mathrm{Ch}$ emMatCARS beamline ID-15-C at the Advanced Photon Source, Argonne, Illinois, USA using an x-ray energy of 11.7 $\mathrm{keV}$. Measurements utilized a 2D PILATUS area detector. The atomic scale surface structure of the liquid is characterized along the surface normal and in plane of the surface by $\mathrm{x}$-ray reflectivity and grazing incidence diffraction (GID), respectively. Diffuse scattering is used to characterize thermal capillary waves. Analysis of the liquid surface reflectivity and diffuse scattering data was performed according to previously published work. ${ }^{6,10}$ 

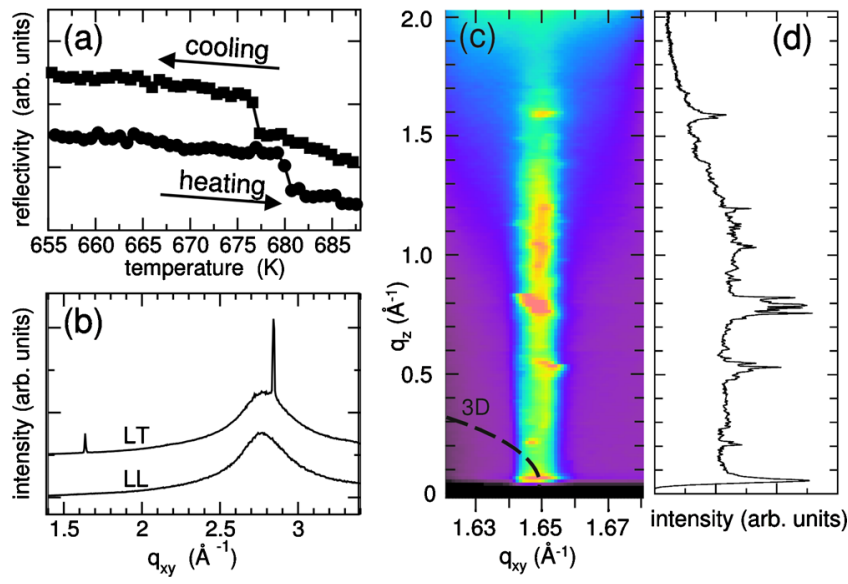

FIG. 1. (Color online) (a) Reflectivity of the liquid $\mathrm{Au}_{49} \mathrm{Cu}_{26.9} \mathrm{Si}_{16.3} \mathrm{Ag}_{5.5} \mathrm{Pd}_{2.3}$ sample at a fixed $q_{z}=1.4 \AA^{-1}$ during heating and cooling of the liquid sample at a rate of about $2 \mathrm{~K} / \mathrm{min}$. (b) GID patterns of the LT surface phase $(T=670 \mathrm{~K})$ and of the LL surface phase $(T=685 \mathrm{~K})$. (c) Crystal truncation rod within the $q_{x y}-q_{z}$ plane of the Bragg reflection of the LT phase at $q_{z}=1.649 \AA^{-1}$ taken by an area detector, showing that the rod is oriented along the surface normal, $z$. The dashed line indicates the shape of the Debye-Scherrer diffraction pattern expected for a 3D powder phase with $q_{x y}^{2}+q_{z}^{2}=(1.649)^{2} \AA^{-2}$. (d) Integrated intensities of the truncation rod data shown in (c).

Figure 1(a) shows the temperature dependence of reflectivity from the surface of liquid $\mathrm{Au}_{49} \mathrm{Cu}_{26.9} \mathrm{Si}_{16.3} \mathrm{Ag}_{5.5} \mathrm{Pd}_{2.3}$ at a fixed angle of $\alpha \equiv \beta=6.78^{\circ}$, corresponding to a momentum transfer of $q_{z}=1.4 \AA^{-1}$. Evidence for a first-order transformation between two different surface phases is shown by the abrupt drop in reflectivity by about $10 \%$ at $680 \mathrm{~K}$ on heating followed by its reversal at about $677 \mathrm{~K}$ on cooling. The apparent hysteresis is probably due to the temperature lag of the thermocouple. No further phase transitions were observed during cooling to temperatures of about $30 \mathrm{~K}$ below $T_{e}$, i.e., in the slightly undercooled region of the liquid.

Figure 1(b) displays GID curves of the liquid sample below and above the surface phase transition temperature, i.e., at 670 and $685 \mathrm{~K}$. These curves correspond to the two surface phases that were revealed by the reflectivity measurements shown in Fig. 1(a). The LL curve, corresponding to $685 \mathrm{~K}$ only shows a broad diffuse maximum centered around $q_{z}=2.75 \AA^{-1}$ that is characteristic of a LL surface phase. In contrast, for the LT phase $(\mathrm{T}=670 \mathrm{~K})$ two sharp Bragg reflections are superimposed on the diffuse maxima of the underlying liquid phase, indicating the presence of in-plane long range, crystalline order at the surface.

Figure 1(c) displays the intensity distribution within the $q_{x y}-q_{z}$ plane of the Bragg reflection at $q_{x y}=1.649 \AA^{-1}$ for the LT phase. The variation in intensity, i.e., the bright spots within the truncation rod as well as the corresponding $q_{z}$ dependence of the $q_{x y}-q_{z}$ integral of the rod intensity that is shown in Fig. 2(d) arises from the superposition of intense diffraction signals from a few large domains on the smooth truncation rod signal from the 2D powder of small sized crystals. The alignment of the truncation rod is along the $q_{z}$ direction, indicating that the surface phase is a $2 \mathrm{D}$ phase. Furthermore, the structureless shape of the smooth portion of the rod intensity as a function of $q_{z}$ demonstrates that the crystals are a monolayer phase, although the $2 \mathrm{D}$ unit cell is not necessarily confined to a single plane. For comparison, truncation rod data that were observed for the LT bilayer phase of liquid $\mathrm{Au}_{82} \mathrm{Si}_{18}$ exhibited a destructive interference
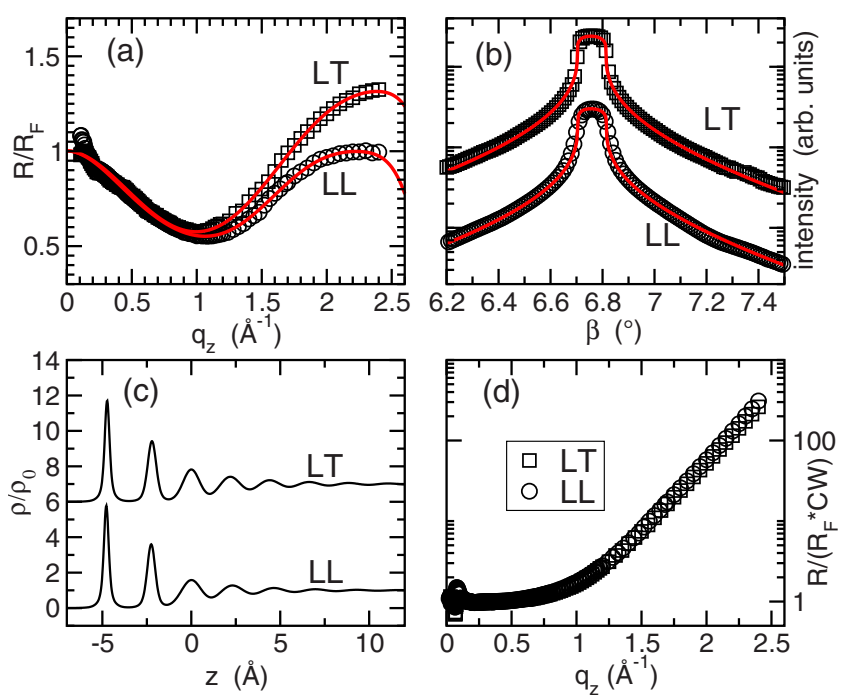

FIG. 2. (Color online) (a) Normalized reflectivity $R / R_{F}$ and (b) diffuse scattering at small angles $\beta$ away from the specular condition $(\alpha \equiv \beta$ $=6.78^{\circ}$ and $q_{z}=1.4 \AA^{-1}$ ) of the two different surface phases LT and LL of liquid $\mathrm{Au}_{49} \mathrm{Cu}_{26.9} \mathrm{Si}_{16.3} \mathrm{Ag}_{5.5} \mathrm{Pd}_{2.3}$. The solid lines are best fits using the model of the electron density normal to the surface shown in (c) and the theoretical cross-sections (Ref. 7). (d) The surface structure factor $\left|\Phi\left(q_{z}\right)\right|^{2}$ that is obtained by dividing the $R / R_{F}$ data for LT and LL phases by the capillary wave contribution $C W$, respectively.

minimum at a position of $q_{z}=\pi / d \approx 0.95 \AA^{-1}$, where $d$ is the distance between the layers.

The reflectivity for the LT and LL phases are shown in Fig. 2(a) in the form of $R\left(q_{z}\right) / R_{F}\left(q_{z}\right)$, where $R_{F}\left(q_{z}\right)$ is the theoretical Fresnel reflectivity for a flat surface with a homogeneous bulk electron density of the $\mathrm{Au}_{49} \mathrm{Cu}_{26.9} \mathrm{Si}_{16.3} \mathrm{Ag}_{5.5} \mathrm{Pd}_{2.3}$ alloy. Note that as $q_{z}$ increases, the reflectivity of the LL surface decreases relative to that of the LT surface. As will be shown in the following, this difference can primarily be understood in terms of the difference between the surface Debye-Waller factor arising from thermally excited surface capillary waves for the two phases.

It is known that the normalized reflectivity $R / R_{F}$ of a liquid surface can be expressed by the surface structure factor which is a functional of the electron density normal to the surface $^{10}$ and a wave vector dependent capillary wave factor, $C W\left(q_{z}\right)$, that arises from thermal height-height correlations of the surface si-23 $^{21}$

$$
\begin{aligned}
& R\left(q_{z}\right) / R_{F}\left(q_{z}\right)=C W\left(q_{z}\right) \times\left|\Phi\left(q_{z}\right)\right|^{2}, \\
& C W\left(q_{z}\right)=\int_{A_{q_{x y}}} d^{2} q_{x y}\left(\frac{q_{x y}}{q_{\max }}\right)^{\eta} \frac{\eta}{2 \pi q_{x y}^{2}} .
\end{aligned}
$$

The capillary exponent is $\eta=\left(k_{B} T / 2 \pi \gamma\right) q_{z}^{2}$, where $\gamma$ is the surface tension. The value $q_{\max }$ is the high frequency cut-off wave vector of capillary waves that for a LL surface is determined by the atomic diameter $d_{a t}, \quad q_{\max }=\pi / d_{a t}$ $=1.16 \AA^{-1}$ with $d_{a t}=2.70 \AA$ for $\mathrm{Au}$ and $\mathrm{Cu}$ as the main components of the alloy. On the other hand, for liquid $\mathrm{Au}-\mathrm{Si}$ the $2 \mathrm{D}$ crystalline surface order introduces surface bending rigidity $\kappa$ that suppresses short wavelength capillary waves. This effect, which reduces the amplitude of the capillary wave factor [Eq. (1)], can be accounted for by replacing $q_{\max }$ with a smaller elastic cut-off wave vector $q_{e}=\sqrt{\gamma / \kappa}$ (Refs. 6 and 24) for the crystalline LT surface phase in Eq. (2). 
One of the standard approaches by which the electron density profile that defines the surface structure factor can be modeled is to use a modified distorted crystal model (DCM) ${ }^{6,10,25}$ For the analysis of the present system an additional sharp first adlayer is added to a slightly modified DCM. A second subtler modification that was required in order to obtain the best possible fits of the reflectivity data [Fig. 2(a)] and the diffuse scattering measured at small angles off the specular condition [Fig. 2(b)] of the LT and LL phases involved the position and amplitude of the second layer of the modified DCM. These effects are hardly visible in the layer that is near $z=-2.5 \AA$ for the best fit profiles that are shown in Fig. 2(c).

The fits obtained similar values of $900 \mathrm{mN} / \mathrm{m}$ for the surface tension of both phases. For the LT phase the best fit value of $q_{e} \approx 0.6 \AA^{-1}$ corresponds to a bending rigidity $\kappa$ $=\gamma / q_{e}^{2} \approx 3 k_{B} T$ of the $2 \mathrm{D}$ crystalline monolayer. For the LL surface phase the best fit was obtained using $q_{\max }$ $=1.16 \AA^{-1}$. The resulting electron density profiles that are shown in Fig. 2(c) and consequently also the corresponding surface structure factors in Fig. 2(d) of the LT and the LL surface phases differ only slightly.

As was also observed for liquid Au-Si (Ref. 6) the difference in the reflectivity between the LT surface containing the monolayer crystals and the LL surface in Fig. 2(a) mainly arises from the bending rigidity of the surface crystals that reduces the amplitude of the capillary wave Debye-Waller factor in Eq. (1). In other words, the rigidity of the surface crystals reduces the amplitude of the small wavelength Fourier components of the surface roughness. This reduction in roughness leads to an increase in reflectivity.

The atomic structure of the monolayer phases of $\mathrm{Au}-\mathrm{Si}$ and $\mathrm{Au}-\mathrm{Cu}-\mathrm{Si}-\mathrm{Ag}-\mathrm{Pd}$ could not be fully resolved within this study. The ratio of $\sqrt{3}$ between the positions of the Bragg reflections of the LT phase in Fig. 1(b) suggests a hexagonal lattice. However, the combination of the missing (20) Bragg reflection that is expected at $q_{x y}=3.298 \AA^{-1}$ and the fact that the corresponding $3.8 \AA$ unit cell dimension is larger than the atomic size suggest a multiatom unit cell.

On passing we note that the atomic layering at the respective LL surface phase of the two glass forming $\mathrm{Au}-\mathrm{Si}$ based alloys is much more pronounced than in the liquid $\mathrm{Au}_{72} \mathrm{Ge}_{28}$ alloy ${ }^{10}$ which is not a glass forming system. ${ }^{19} \mathrm{We}$ can speculate that the liquid structure of $\mathrm{Au}-\mathrm{Cu}-\mathrm{Si}-\mathrm{Ag}-\mathrm{Pd}$ has a high degree of order that is comparable to what is found in the corresponding glass phase. ${ }^{26}$ Consequently, it will contain icosahedral-like order and clusters similar to $\mathrm{Au}-\mathrm{Si}$ (Refs. 5 and 16) or other glass forming alloys with multishell cluster sizes. ${ }^{27}$

We can also draw attention to the fact that the surface induced layering of the Au-Si-based alloys is substantially more pronounced than for the other liquid metals, e.g., liquid $\mathrm{Sn},{ }^{28} \mathrm{Ga},{ }^{29}$ eutectic Bi-Sn (Ref. 30) and In-Bi. ${ }^{31}$ None of the latter liquids are known to exhibit either glass formation during quenching or surface crystallization of the liquid phase.

We, therefore, propose that the high degree of icosahedral-like order in the bulk liquid, which is responsible for glass forming ability, also plays an important role in obtaining a high degree of layering at the liquid/vapor interface, i.e., a high degree of order normal to the surface as well as in a increased tendency for formation of crystalline sur- face frozen phases. This implies a different origin of surface freezing in these than in the phase separated Ga-based alloys. ${ }^{89}$ It would be reasonable to suspect that surface crystallization should occur in many other metallic liquids that exhibit a high degree of order in the bulk phase. This would include both strong glass forming liquids and icosahedral quasicrystal forming liquids. Since it is known that local icosahedral ordering greatly increases during undercooling of metallic liquids ${ }^{13}$ surface crystallization may be an important property in direct near net shape casting of metallic microparts and molding of metallic glasses. In particular, this could occur for parts with dimensions in the nanometer range where the wetting behavior of the liquid on the crucible was found to be crucial. ${ }^{12}$

Research supported by the U.S. Department of Energy (DOE), Office of Basic Energy Sciences, Division of Materials Sciences and Engineering under Award No. DE-FG0288ER45379. ChemMatCARS is principally supported by the National Science Foundation/DOE under Grant No. NSF/ CHE-0822838. The Advanced Photon Source is supported by the U.S. DOE, Basic Energy Sciences, Office of Science, under Contract No. W-31-109-Eng-38. S.M. acknowledges support by the German Research Foundation through Grant No. Me 3113/2-2. Discussions with Oleg Shpyrko (UCSD) are gratefully acknowledged. The authors thank Sven E. Stoltz for assistance during the experiment.

${ }^{1}$ M. P. D’Evelyn and S. Rice, Phys. Rev. Lett. 47, 1844 (1981).

${ }^{2}$ O. G. Shpyrko, et al. Science 313, 77 (2006).

${ }^{3}$ O. G. Shpyrko, et al. Phys. Rev. B 76, 245436 (2007).

${ }^{4}$ A. L. Pinardi, S. J. Leake, R. Felici, and I. K. Robinson, Phys. Rev. B 79, 045416 (2009).

${ }^{5}$ T. U. Schülli, et al. Nature (London) 464, 1174 (2010).

${ }^{6}$ S. Mechler, et al. Phys. Rev. Lett. 105, 186101 (2010).

${ }^{7} \mathrm{~S}$. Mechler, et al. (to be published).

${ }^{8}$ B. Yang, et al. Proc. Natl. Acad. Sci. U.S.A. 96, 13009 (1999).

${ }^{9}$ B. Yang, D. Li, and S. A. Rice, Phys. Rev. B 67, 212103 (2003).

${ }^{10}$ P. S. Pershan, et al., Phys. Rev. B 80, 125414 (2009).

${ }^{11}$ W. Klement, R. H. Willens, and P. Duwez, Nature (London) 187, 869 (1960).

${ }^{12}$ G. Kumar, H. X. Tang, and J. Schroers, Nature (London) 457, 868 (2009).

${ }^{13}$ Y. T. Shen, T. H. Kim, A. K. Gangopadhyay, and K. F. Kelton, Phys. Rev. Lett. 102, 057801 (2009).

${ }^{14} \mathrm{H}$. W. Sheng et al., Nature (London) 439, 419 (2006).

${ }^{15}$ S. Mechler et al., Phys. Rev. B 81, 180101 (2010).

${ }^{16}$ A. Pasturel, E. S. Tasci, M. H. F. Sluiter, and N. Jakse, Phys. Rev. B 81 140202 (2010).

${ }^{17}$ S. Mechler, G. Schumacher, V. Koteski, H. Riesemeier, F. Schäfers, and H. E. Mahnke, Appl. Phys. Lett. 97, 041914 (2010).

${ }^{18}$ F. C. Frank, Proc. R. Soc. London, Ser. A 215, 43 (1952).

${ }^{19}$ P. Ramachandrarao and T. R. Anantharaman, Trans. Metall. Soc. AIME 245, 886 (1969).

${ }^{20}$ J. Schroers, B. Lohwongwatana, W. L. Johnson, and A. Peker, Appl. Phys. Lett. 87, 061912 (2005).

${ }^{21}$ S. K. Sinha, E. B. Sirota, S. Garoff, and H. B. Stanley, Phys. Rev. B 38 2297 (1988)

${ }^{22}$ F. P. Buff, R. A. Lovett, and F. H. Stillinger, Phys. Rev. Lett. 15, 621 (1965)

${ }^{23}$ P. S. Pershan, Colloids Surf., A 171, 149 (2000).

${ }^{24}$ H. Kellay and J. Meunier, J. Phys.: Condens. Matter 8, A49 (1996).

${ }^{25}$ O. M. Magnussen et al., Phys. Rev. Lett. 74, 4444 (1995).

${ }^{26}$ X. D. Wang, et al., Scr. Mater. 64, 81 (2011).

${ }^{27}$ D. B. Miracle, Nature Mater. 3, 697 (2004).

${ }^{28}$ O. G. Shpyrko et al., Phys. Rev. B 70, 224206 (2004).

${ }^{29}$ M. J. Regan, et al., Phys. Rev. Lett. 75, 2498 (1995).

${ }^{30}$ O. G. Shpyrko et al., Phys. Rev. Lett. 95, 106103 (2005).

${ }^{31}$ E. DiMasi et al., Phys. Rev. Lett. 86, 1538 (2001). 